\title{
Representaciones "positivas" y "negativas" sobre el éxtasis en un grupo de consumidores en Coimbra (Portugal)
}

\author{
Fernando J. F. Mendes*; Lurdes LombA** \\ * Psicologo Clínico. Presidente Irefrea Portugal \\ * Escola Superior de Enfermagem de Coimbra \\ Enviar correspondencia a: \\ Lurdes Lomba. Escola Superior de Enfermagem de Coimbra.Rua 5 de Outubro, Apartado 55. 3001-901 Coimbra. Portugal. Correo-e: irefrea@netcabo.pt \\ Recibido: Agosto de 2006 \\ Aceptado: Junio de 2007
}

\section{RESUMEN}

Se estudian las representaciones 'positivas' y negativas' que tenían sobre el éxtasis una muestra de 223 jóvenes consumidores de éxtasis en la ciudad de Coimbra, reclutados utilizando la técnica de bola de nieve. En el ámbito de las representaciones negativas reseñadas destacan los efectos nocivos sobre el cerebro y entre los positivos destaca el placer asociado al baile. Los consumidores valoran las representaciones negativas sobre esta sustancia pero dan mucha más importancia a las representaciones positivas. La valoración de las representaciones positivas no varía en función de que los consumos sea más o menos frecuentes, se tomen un mayor o menor número de pastillas o si se hace policonsumo. Sin embargo aquellos jóvenes con representaciones sobre el éxtasis más negativas tienen un patrón de consumo menos abusivo. Se discuten las implicaciones preventivas.

Palabras clave: éxtasis/MDMA, representaciones positivas, representaciones negativas.

\section{ABSTRACT}

The positive and negative representations of ecstasy were studied in a sample of 223 young people who consumed ecstasy in the city of Coimbra, recruited using the snowball technique. With regard to negative representations they highlight the harmful effects on the brain, with regard to positive effects they highlight the pleasure associated with dancing. The consumers acknowledge the negative representations but consider the positive representations much more important. Rating of the positive representations does not vary according to frequency of consumption, number of pills consumed or whether or not they are polyconsumers. However, those with more negative representations show less abusive consumption patterns. Implications for prevention are discussed.

Key words: ecstasy/MDMA, positive representations, negative representations.

\section{INTRODUCCIÓN}

D e acuerdo con el OEDT (2006) la segunda sustancia ilegal más consumida en Europa es el grupo de las drogas sintéticas, entre las que está el éxtasis o MDMA como la más frecuente. Según la mima fuente el consumo de esta sustancia entre la población general es habitualmente bajo $(0,8 \%$ de la población portuguesa), pero estas frecuencias obviamente suben si nos referimos a poblaciones más jóvenes (IDT, 2005). El consumo de éxtasis llega a ser particularmente alto en contextos recreativos, de forma que dichos ambientes pueden llegar a ser considerados como factores de riesgo (Bellis, Hughes, Bennett y Thomson, 2003). Por esta razón, Calado (2006) denominó a estas sustancias como las "drogas del futuro". De hecho, tal como refiere Lomba (2006) este tipo de drogas se diferencia de las restantes por su especial conexión con las actividades recreativas de fin de semana, por introducir nuevas formas de diversión, nuevos valores estéticos, condicionan las diferentes formas de comunicación y determinan nuevos patrones de consumo.

El uso recreativo de éxtasis es una realidad de la cultura recreativa nocturna de fin de semana pero, para la comprensión del fenómeno no basta con conocer las frecuencias de consumo y otros datos sociodemográficos sino que también interesa conocer el proceso de motivación subyacente al consumo así como las representaciones que los individuos construyen acerca del éxtasis pues son importantes elementos caracterizadores que "nos ayudan a pensar y a actuar en relación a la droga" (Valentim, 1998, p. 56).

Así, uno de los aspectos a tener en cuenta son las representaciones que se tienen sobre las sustancias 
por parte de los usuarios. Según Negreiros (2000) citando a Farr y Moscovici, las representaciones son un "conjunto de cogniciones ou de universos de opiniones compartidos por un grupo amplio de individuos, acerca de temas específicos del ambiente social", en este caso, las drogas. Estas representaciones son como una forma de conocimiento práctico, que acaba por generar, modelar y determinar los comportamientos de consumo, definiendo la naturaleza de los estímulos que rodean el consumo y el significado que dan a los efectos del consumo de las sustancias (Oliveira, 2001).

Las representaciones, se aprenden a través de procesos cognitivos, pero también a través de procesos de motivación que orientan la dinámica de esos procesos cognitivos (Vala, 1997). Además las representaciones pueden ser sometidas a distorsiones, esto es a sobrevaloraciones o desvaloraciones de sus atributos, pero también a la supresión o a la adición de propiedades que no le pertenecen (Costa y Marques, 2002). El conocimiento de estos mecanismos y de los factores que los influencian es una premisa fundamental para la comprensión del consumo tal como es y puede ser útil para una intervención preventiva efectiva.

De hecho, analizando alguno de los estudios publicados en el ámbito de las representaciones de sustancias psicoactivas (Castellana y Lladó,1999; Zupiria, González y Yllá,1999; Bejerano, Lee y Ugalde, 1999) podemos ver que las expectativas que se poseen sobre una sustancia adictiva están relacionadas con el consumo posterior, llegándose a corroborar que las expectativas son predictivas del consumo. Otras investigaciones que tratan de identificar los diferentes factores que influencian el inicio de la conducta adictiva proponen que seria necesario intervenir sobre el conjunto de creencias si queremos actuar preventivamente (Castro y Godoy, 1999; Peral y Caballero-Reales, 1999). Esto es, están aludiendo a la representación, en cuanto conjunto de creencias, como elemento clave a tratar en las propuestas de prevención.

En el presente estudio se pretende conocer más sobre las representaciones que sobre el éxtasis tienen una muestra de usuarios de esta sustancia en la ciudad de Coimbra (Portugal), junto con otros datos como patrones de consumo, etc. Se utilizó, para ello un cuestionario adaptado del "Questionnaire of social representations of ecstasy"(Calafat, Stocco, Mendes, Simon, Wijngaart, Sureda et al, 1998), y que ya fue utilizado en una muestra de usuarios de éxtasis en Coimbra. El estudio intenta establecer la relación entre patrones de consumo y las representaciones que se tienen sobre dicha sustancia.

\section{MATERIAL Y MÉTODOS}

El estudio se realizó en Coimbra de enero a junio de 2003 entre jóvenes consumidores de 13 a 24 años, a una muestra de 223 consumidores de éxtasis, que fueron entrevistados recurriendo a la técnica de muestreo en bola de nieve (Fortín, 1999). Con la colaboración de DJ's y otros profesionales que trabajaban en bares y discotecas se identificaron un primer grupo de jóvenes consumidores de éxtasis. A partir de estas 'semillas' se fueron haciendo otros contactos entre sus conocidos y amigos, y así sucesivamente. De esta forma fueron distribuidos 300 cuestionarios que debían ser rellenados por los propios jóvenes. Se recogieron 250, y de estos 37 fueron eliminados por estar incompletos o por corresponder a individuos mayores de 25 años.

El 'patrón de consumo de éxtasis' tiene tres categorías: excesiva, habitual y ocasional. Estas tres categorías se definen a partir de las variables 'frecuencia de consumo', 'número de comprimidos que toman cada vez' y 'policonsumo' de otras drogas.

En cuanto a las 'frecuencias de consumo' se utilizan criterios similares a los de Calafat et al. (1998) para subdividir la muestra. Se considera consumo ocasional los que han consumido una vez o ninguna durante el último mes. Algunas veces al mes pero menos de una vez por semana y una vez por semana se consideran que definen el consumo habitual. Por último, algunas veces por semana y casi todos los días se considerarían consumo excesivo.

En cuanto a 'número de comprimidos que toma cada vez' consideramos que un consumo de "media pastilla" por ocasión es compatible con el consumo ocasional, mientras que 'una pastilla' caracteriza un consumo habitual y un consumo de "más de 1 pastilla" indica un consumo excesivo, tal como refiere la OEDT (2001).

El "policonsumo" se define como ocasional cuando el joven refiere consumir alcohol y/o tabaco simultáneamente con el éxtasis. Hablamos de habitual cuando el consumo de éxtasis (con o sin consumo de alcohol y tabaco) es simultáneo con el de cannabis. La OEDT (2002) considera que el patrón de policonsumo más frecuente con drogas estimulantes es la asociación con el cannabis y alcohol. Por otro lado debemos tener en cuenta que el cannabis es la droga ilegal con mayor prevalencia de consumo entre la población portuguesa (Balsa, Farinha, Urbano y Francisco, 2001). Definimos como excesivo el consumo simultáneo de éxtasis con otras drogas ilegales que no sean el cannabis (aunque lógicamente lo pueden haber consumido).

En lo referente a las representaciones que los jóvenes tienen del éxtasis fueron medidas recurriendo a una escala de análisis construida para ese efecto. Se trata del "Questionnaire of social representations of 
ecstasy", que ya hemos citado (Calafat et al., 1998). La escala se presenta con dos dimensiones. Hay una "subescala" de representaciones positivas, con 17 propuestas referente a las expectativas positivas de la acción del éxtasis, que cubre los efectos hedónistas, psicológicos y estimulantes de la droga. La "subescala" de representaciones negativas tiene 15 propuestas, que reflejan la evaluación que los jóvenes hacen de su nocividad y de los riesgos asociados a su consumo.

Con cada 'propuesta' (se trata de una frase sobre un supuesto efecto del éxtasis) se puede estar: 'en desacuerdo total', 'algo en desacuerdo', 'ni de acuerdo ni en en desacuerdo', 'de acuerdo' y 'de acuerdo totalmente'. Se puntuaba cada respuesta de 1 a 5 puntos, correspondiendo el mínimo a la primera y el máximo a la última. El resultado final de la suma de las puntuaciones puede variar en el caso de la subescala positiva entre los $17(1 \times 17)$ y los $85(5 \times 17)$ puntos. En el caso de la sub-escala de las representaciones negativas las puntuaciones variarían entre los $15(1 \times 15)$ y los 75 (5x15) puntos. En ambas subescalas una menor puntuación corresponde a un menor grado de concordancia y una mayor puntuación corresponde a un mayor de concordancia con las propuestas. Además podemos jerarquizar las representaciones dentro de cada subescala en función de la puntuación que cada item ha recibido.

Se aseguró la validez del contenido de la escala, una vez que éste se basaba en un cuestionario similar, y las alteraciones introducidas se fundamentaban en datos recogidos fruto de una investigación bibliográfica previa.

Se confirmó la fidelidad de la escala verificando la consistencia interna (homogeneidad de los ítems) para cada una de las dimensiones. Para ello se calculó el $\alpha$ de Cronbach, cuyo valor más bajo encontrado fue de 0,79 , lo que indica una fiabilidad satisfactoria. Se evaluó también la consistencia interna de los ítems para cada dimensión (coeficiente de correlación corregido entre el resultado en el ítem y el resultado en la escala). Se verificó que todos los ítems presentaban correlaciones aceptables con el total de la escala, siendo el valor de la correlación más bajo de 0,25. Por tanto, se decidió mantener el conjunto inicial de los 32 ítems.

Para el estudio de la validez del constructo, utilizamos el análisis factorial en componentes principales (ACP) para la totalidad de los 32 ítems, usando como criterio para la retención de los factores la presentación de valores propios superiores a 1. De este modo, fueron extraídos dos factores que fueron rodados ortogonalmente usando el procedimiento VARIMAX. Del análisis de la matriz factorial se concluyó que todos los ítems saturan en la dimensión definida conceptualmente, no saturando ningún ítem en más que un factor con peso > 0,3, lo que abona en favor de la validez del constructo de este instrumento.

\section{RESULTADOS}

\section{Descripción de los consumidores de éxtasis}

Se trata de un grupo de jóvenes socialmente integrados, en que la mayoría estudia $(50,7 \%)$ o trabaja (37,2\%). La edad media es de 21 años y predomina el sexo masculino $(70,4 \%)$, los solteros $(91,9 \%)$; la mayoría $(66,4 \%)$ viven con sus familiares.

Son jóvenes que frecuentan asiduamente cafés $(56,0 \%)$, bares $(30,0 \%)$, discotecas $(30,4 \%)$ y after hours $(30,9 \%)$. Casi todos los amigos de estos jóvenes consumen alcohol $(87,4 \%)$, tabaco $(91,5 \%)$ o cannabis $(83,8 \%)$, la mayoría consume éxtasis $(59,1 \%)$ y algunos consumen cocaína $(46,2 \%)$. En relación a los padres de estos jóvenes el 11,7\% consumen alcohol en exceso y el 8,5\% consumen drogas ilegales. En los jóvenes de este estudio, como suele ser habitual, el consumo ocurre de preferencia en fin de semana $(85,2 \%)$, en ambientes de música y baile (fiestas: $86,1 \%$ y discotecas: $71,7 \%$ ) y en un contexto grupal, con los amigos (91,9\%). Hay una fuerte adhesión de estos jóvenes a la música House (83,9\%) y se verificaron relaciones positivas $(p<.005)$ de consumo con los subtipos Tecno y Trance. Cuando se les preguntó si pensaban abandonar el consumo de éxtasis casi la mitad (49,3\%) dicen que piensan mantenerlo mientras que la otra mitad $(50,7 \%)$ piensa en ir dejándolo.

\section{Patrones de consumo}

Se han tenido en cuenta tres patrones de consumo: ocasional, habitual y excesivo (ver Tabla 1). Podemos ver esta distribución según tres variables. En relación con la variable "frecuencia de consumo" resulta que más de la mitad de la muestra $(53,8 \%)$ son consumi-

Tabla 1 - Distribución de los jóvenes según su patrón de consumo de éxtasis utilizando como indicadores las variables frecuencia, cantidad (número de comprimidos) y policonsumo $(\mathrm{N}=223)$

\begin{tabular}{|c|c|c|c|}
\hline Patrón de Consumo & Frecuencia (\%) & Cantidad (\%) & Policonsumo (\%) \\
\hline Ocasional & 53,8 & 21,1 & 6,7 \\
Habitual & 34,5 & 19,3 & 35,0 \\
Excesivo & 11,7 & 59,6 & 58,3 \\
\hline
\end{tabular}


dores 'ocasionales', mientras que alrededor de una tercera parte $(34,5 \%)$ son consumidores 'habituales'. En relación a la variable 'número de pastillas por toma' la mayoría de los jóvenes son consumidores "Excesivos", es decir, consumen más de un comprimido en cada ocasión. En relación con el "policonsumo' tenemos que alrededor de una tercera parte $(35,0 \%)$ son consumidores "habituales" y que la mayoría (58,3\%), según esta forma de clasificación, pueden ser considerados consumidores "excesivos".

\section{Representaciones sociales}

Como ya se ha indicado, el estudio permite jerarquizar los ítems más valorados por los jóvenes en ambas dimensiones de la escala, atendiendo a la concordancia que los jóvenes demuestran con sus respuestas en cada ítem. Así, en el ámbito de las representaciones positivas, los ítems más puntuados se relacionan con el placer del baile, el bienestar consigo mismo y con los otros (Tabla 2).En el ámbito de las representaciones negativas los más valorados son los efectos nocivos del éxtasis a nivel cerebral y a nivel legal (Tabla 3).

\section{Relación entre patrón de consumo y representa- ciones del éxtasis}

Un objetivo de esta investigación era averiguar si había relación entre el patrón de consumo del éxtasis y las representaciones del éxtasis (ver Tabla 4). Las representaciones positivas de los jóvenes no son estadísticamente diferentes según su patrón de consumo de éxtasis, bien se trate de la variable frecuencia, cantidad o policonsumo, mientras que sí existían diferencias significativas $(p=0,001)$ para las representaciones negativas si consideramos la variable frecuencia de consumo de éxtasis de los jóvenes. Con la realización del test Post Hoc de Turkey para análisis de la diferencia intergrupos, se observó que los jóvenes que consumen éxtasis con una frecuencia habitual tienen representaciones del éxtasis menos negativas que los jóvenes que consumen ocasionalmente.

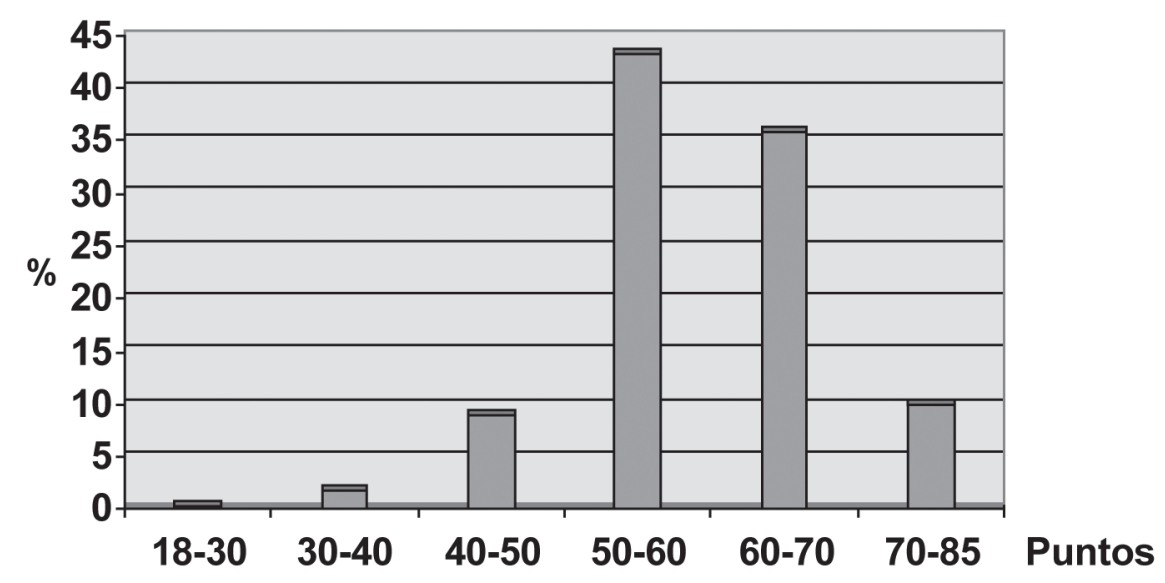

Figura 1: Distribución de los jóvenes de acuerdo a la puntuación obtenida en la subescala de las representaciones positivas del éxtasis

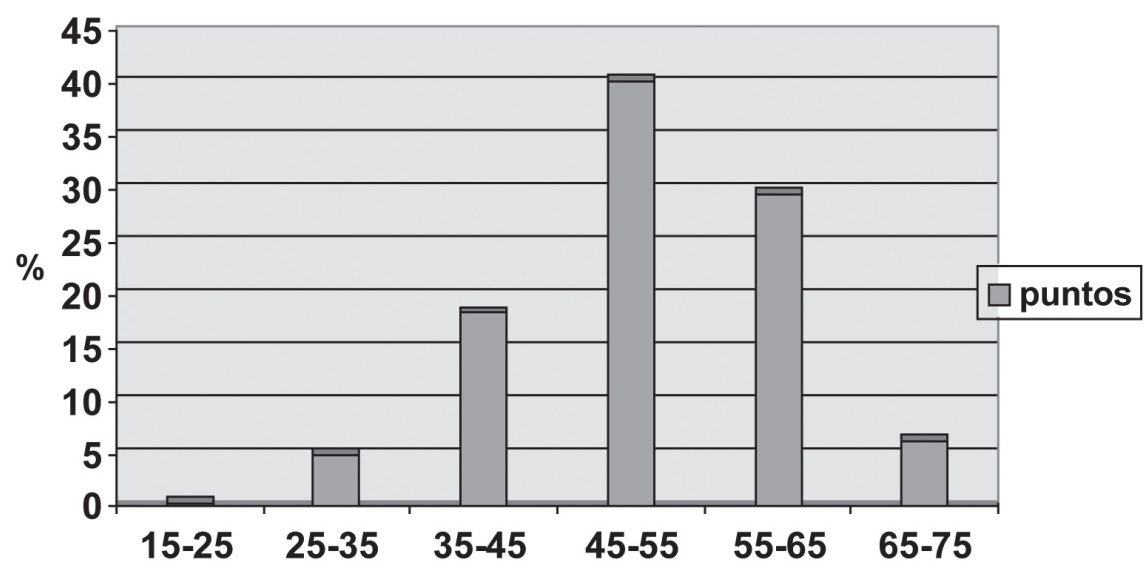

Figura 2: Distribución de los jóvenes de acuerdo a la puntuación obtenida en la subescala de las representaciones negativas del éxtasis. 
Tabla 2 - Distribución de las representaciones positivas del éxtasis de acuerdo a la suma de las puntuaciones obtenidas.

\begin{tabular}{|l|c|}
\hline ITEMS DE LAS REPRESENTACIONES POSITIVAS & Suma de las Puntuaciones \\
\hline El éxtasis aumenta el placer de bailar & 963 \\
El éxtasis permite bailar durante más tiempo & 955 \\
El éxtasis nos hace sentir eufóricos y felices & 927 \\
El éxtasis nos hace sentir bien & 881 \\
El éxtasis mantiene a las personas despiertas & 867 \\
El éxtasis ayuda a establecer relaciones y nos sentimos bien con los otros & 836 \\
El éxtasis estimula los sentidos & 831 \\
El éxtasis tiene un precio accesible & 803 \\
El éxtasis desinhibe sexualmente & 772 \\
El éxtasis permite comprender la música House, techno,... & 763 \\
El éxtasis es bueno para evadirnos de la realidad, olvidar los problemas & 761 \\
El éxtasis mejora el sexo & 761 \\
El éxtasis estimula la creatividad & 760 \\
El éxtasis es una solución para personas tímidas & 727 \\
El éxtasis relaja & 653 \\
El éxtasis es una droga limpia, fácil de usar y confortable & 612 \\
El éxtasis pone las ideas en orden & 470 \\
\hline
\end{tabular}

\section{Tabla 3 - Distribución de las representaciones negativas del éxtasis de acuerdo a la suma de las} puntuaciones obtenidas.

\begin{tabular}{|l|c|}
\hline ITEMS DE LAS REPRESENTACIONES POSITIVAS & Suma de las Puntuaciones \\
\hline El éxtasis aumenta el placer de bailar & 963 \\
El éxtasis permite bailar durante más tiempo & 955 \\
El éxtasis nos hace sentir eufóricos y felices & 927 \\
El éxtasis nos hace sentir bien & 881 \\
El éxtasis mantiene a las personas despiertas & 867 \\
El éxtasis ayuda a establecer relaciones y nos sentimos bien con los otros & 836 \\
El éxtasis estimula los sentidos & 831 \\
El éxtasis tiene un precio accesible & 803 \\
El éxtasis desinhibe sexualmente & 772 \\
El éxtasis permite comprender la música House, techno,... & 763 \\
El éxtasis es bueno para evadirnos de la realidad, olvidar los problemas & 761 \\
El éxtasis mejora el sexo & 761 \\
El éxtasis estimula la creatividad & 760 \\
El éxtasis es una solución para personas tímidas & 727 \\
El éxtasis relaja & 653 \\
El éxtasis es una droga limpia, fácil de usar y confortable & 612 \\
El éxtasis pone las ideas en orden & 470 \\
\hline
\end{tabular}

Tabla 4: Síntesis de los resultados estadísticos obtenidos en la prueba ANOVA entre el patrón de consumo de éxtasis y las representaciones que los jóvenes tienen del éxtasis.

\begin{tabular}{|c|c|c|c|c|c|c|c|c|}
\hline \multirow{3}{*}{ Representaciones } & \multicolumn{6}{|c|}{ Patrón de Consumo } & \multirow{3}{*}{$\mathrm{F}$} & \multirow{3}{*}{$\mathrm{p}$} \\
\hline & \multicolumn{2}{|c|}{ Ocasional } & \multicolumn{2}{|c|}{ Habitual } & \multicolumn{2}{|c|}{ Excesivo } & & \\
\hline & $\overline{\mathrm{X}}$ & DT & $\bar{X}$ & DT & $\bar{X}$ & DT & & \\
\hline \multicolumn{9}{|c|}{ Frecuencia } \\
\hline Positivas & 58,79 & 8,69 & 60,69 & 8,98 & 62,08 & 9,44 & 2,014 & 0,136 \\
\hline Negativas & 53,41 & 9,39 & 49,18 & 9,34 & 50,42 & 8,90 & 5,058 & $0,007^{*}$ \\
\hline \multicolumn{9}{|c|}{ Cantidad } \\
\hline Positivas & 57,19 & 9,17 & 59,77 & 8,65 & 60,78 & 8,78 & 2,865 & 0,059 \\
\hline Negativas & 54,40 & 8,56 & 50,70 & 11,16 & 50,90 & 9,10 & 2,645 & 0,073 \\
\hline \multicolumn{9}{|c|}{ Policonsumo } \\
\hline Positivas & 55,20 & 11,13 & 59,47 & 7,91 & 60,58 & 9,10 & 2,577 & 0,078 \\
\hline Negativas & 55,40 & 6,46 & 51,08 & 9,59 & 51,48 & 9,68 & 1,336 & 0,265 \\
\hline
\end{tabular}

*Diferencia significativa para $p<0,05$. 


\section{DISCUSIÓN}

Comparando los resultados de este estudio con la muestra utilizada en el estudio de Calafat et al (1998), también realizado en Coimbra, concluimos que los jóvenes se están iniciando más precozmente en el consumo (17,8 años cuando en el estudio anterior eran 21,3$)$, están consumiendo mayor cantidad de éxtasis $(59,6 \%$ tienen actualmente consumos superiores a 1 comprimido, cuando antes eran un 35,2\% los que tomaban más de una pastilla por noche y recurren más al policonsumo (actualmente hay un 89,2\% que toma simultáneamente alcohol cuando antes era un $18,3 \%$ y un $56,5 \%$ que lo combina con cannabis o cocaína cuando antes era un $11,9 \%$ ), aunque es cierto que el consumo concomitante con drogas alucinógenas como el LSD o setas ha disminuido.

Los resultados encontrados apuntan hacia un consumo generalizado de drogas entre el grupo de pares, siendo el consumo de cannabis casi similar al de tabaco o alcohol, lo cual es un gran factor de riesgo. También, como mantiene el SPTT (2002), tienen gran importancia las actitudes permisivas hacia las drogas del grupo de amigos. Esta influencia es tan importante que Botella (2000) destaca las relaciones con los pares como el factor de riesgo más importante, al igual que lo hacen Palmer, Montaño y Calafat (2000). También es un factor de riesgo que un $20,2 \%$ de los padres de estos jóvenes consuman alcohol y otras drogas excesivamente. Como señala Lomba (2006), basándose en distintas investigacione "El uso de drogas por parte de los padres y sus actitudes hacia el alcohol y otras drogas se relaciona con el las actitudes de los adolescentes frente al alcohol y las otras drogas".

El estudio confirma la ya conocida relación entre baile y consumo de éxtasis. Tal como refiere Gallego, Hofstadt, Estévez, López y Castillo ( 2001), a representación que los jóvenes tienen de la droga se asemeja a un caleidoscopio de relaciones sociales, donde los jóvenes la perciben como el común denominador de actividades eminentemente lúdicas como bailar, oír música, ir a discotecas o salir de marcha. La industria recreativa juega muchas veces con esto, empleando los supuestos efectos del éxtasis para la promoción de las actividades recreativas. El uso de adjetivos descriptivos como éxtasis, locura, delirio y hedonista son ejemplos de referencias corrientemente asociadas a "fiestas" que mantienen y legitiman la relación entre diversión y drogas (Calafat, Fernández, Bellis, Montserrat, Mendes, Hughes, et al, 2004).

Después del placer del baile como supuesto efecto del consumo de éxtasis, los jóvenes valoran el sentirse bien consigo mismo y mejorar la relación con los otros como resultado del consumo. También Alarcão (2000) y López (2007) refieren que los jóvenes ven en el consumo de drogas una forma de iniciación y de ser aceptados por el grupo, así como de socialización con los pares, facilitando el sentirse bien y ser aceptado como parte de un grupo. Estos resultados pueden hacer pensar en la necesidad de que los programas preventivos tengan en cuenta estas necesidades básicas de los individuos como pueden ser gustarse a si mismos o sentirse bien en las relaciones que se establecen con los otros, sin que ese placer venga de la manipulación de drogas. Rita (2001) defiende la necesidad de una prevención que se base en la construcción de lazos afectivos fundamentales como los lazos familiares que son el soporte de nuestra construcción individual y social.

Es interesante observar que las representaciones negativas obtienen puntuaciones elevadas, pero, a pesar de ello, éstas son manifiestamente inferiores a las puntuaciones de la escala de las representaciones positivas. Son conscientes de que puede haber riesgos, pero, como suele decirse, las ventajas superan los inconvenientes.

Los estudios recientes do ESPAD (Andersson, Hibell, Beck, Choquet, Kokkevi, Fotiou et al., 2007) sobre el beber concentrado (binge drinking) en Europa también muestran resultados acerca de este equilibrio que establecen los jóvenes sobre las ventajas e inconvenientes del consumir drogas. Aquellos países en los que hay mayor proporción de embriagueces existe mayor conciencia de los problemas derivados del abuso del alcohol, pero también es mayor la percepción de las ventajas que aportan las embriagueces.

Así, a nivel da prevención, no podemos conformarnos con informar a los jóvenes sobre los peligros del consumo de drogas. El problema pasa a ser como conseguir que se valoren menos positivamente los efectos de las drogas, de acuerdo a los resultados de este estudio. Esta no es una cuestión fácil de resolver pues hay que tener cuidado con exagerar las consecuencias y presentar los casos extremos, debido a que pueden provocar escepticismo en los consumidores (Vastag, 2001), aunque no son temas cerrados y seguramente sería oportuno seguir investigando sobre estos temas sobre los que conocemos poco. En nuestro caso, y de acuerdo a nuestros resultados, no basta con alertar de los peligros (puesto que los consumidores ya los tienen en cuenta), sino que se trata de cómo conseguir que el consumo de éxtasis como estrategia personal para conseguir determinados logros sea algo menos valorado.

Los ítems menos puntuados de la subescala de las representaciones negativas fueron "El éxtasis es una droga peligrosa porque lleva a la dependencia" o "Las personas que consumen éxtasis son personas con problemas", lo cual podría apuntar a que no se quieren ver los problemas más crónicos debidos al consumo y que tampoco los consumidores de éxtasis quieren identificarse con personas problemáticas. No en tanto este proceso cognitivo que López (2007) chamou de 
"redefinición del consumo como un problema" es fundamental para que o joven procure acuda para césar el consumo ou estrategias para reducir danos.

Es interesante también que las representaciones positivas sobre el éxtasis no sean diferentes según el patrón de consumo (frecuencia, cantidad o policonsumo). Todos los jóvenes consumidores comparten la misma valoración positiva que Sierra, Pérez, Pérez y Nuñez, (2005) y Camerotti y Kornblit (2005) también han encontrado nos seus estudios y mencionan ser los efectos hedónicos de las substancias, como la diversión, la disminución de sensaciones desagradables, la percepción de baja peligrosidad y creencia de razones de consumo asociadas a la evasión de la realidad. Por tanto, no hemos podido establecer la hipótesis de que los patrones de consumo más elevados estarían relacionados con representaciones más positivas de la sustancia. Tal como refiere Gallego et al., (2001), podemos pensar que el consumo es "alentado culturalmente", es decir dentro del grupo de consumidores se forman argumentaciones que acaban siendo asimiladas transversalmente por todos los tipos de consumidores.

El presente estudio no establece una comparación entre consumidores de éxtasis y no consumidores. Pero si sabemos a partir de otro estudio que utiliza una metodología similar y que si establece la comparación entre consumidores y no consumidores, que estos últimos tienen una visión menos positiva del éxtasis que los consumidores (Calafat et al., 1998). Desde esta perspectiva comparativa con los no consumidores quizás valga la pena el 'deconstruir' con intencionalidad preventiva las representaciones positivas más frecuentes entre los jóvenes como son aquellas que creen que el éxtasis facilitan el baile, la felicidad o las relaciones personales.

Sin embargo si que encontramos diferencias significativas acerca de las representaciones negativas de acuerdo a la frecuencia de consumo ( $p=0,001)$, de tal forma que los jóvenes que consumen éxtasis con más frecuencia tienen representaciones menos negativas del éxtasis. Aquí sí se verifica la esperada modulación de las representaciones, probablemente debido a la familiarización del joven con el éxtasis y subsiguiente disminución de miedos iniciales, así como por la necesidad de pensar que se está en control de la situación. Aquí sí confirmamos la hipótesis y los datos del anterior estudio sobre las representaciones del éxtasis realizada por Calafat et al. (1998) de que el miedo a las consecuencias negativas por el uso del éxtasis tiene cierta influencia sobre la frecuencia de consumo. También podemos pensar como Gallego et al. (2001), que cree que las representaciones que los jóvenes poseen sobre los riesgos de las drogas son un predictor de los consumos.
Este estudio tiene limitaciones puesto que no tiene en cuenta otros factores de riesgo que pueden tener su importancia a la hora de tener ciertas representaciones sobre las drogas o sobre su consumo. Sin embargo, permite verificar que los jóvenes consumidores a pesar de conocer los efectos nocivos del éxtasis, tienen representaciones francamente positivas de la droga, asentadas en el placer, en el bienestar y en el baile. Estas representaciones, fruto de procesos cognitivos y de mecanismos de motivación individuales pero también fruto de procesos de construcción social, favorecen el consumo. Tanto que, los resultados obtenidos que apuntan para que los jóvenes que tienen las representaciones más negativas del éxtasis consumen menos, avalan esta idea de que las representaciones del éxtasis influencian la relación de consumo de los jóvenes con la droga. Como repercusiones tenemos que, mientras las representaciones hedónicas de la droga se sobrepongan a las representaciones asentadas en la percepción del riesgo y en el reconocimiento de los efectos nocivos del éxtasis, el consumo se justifica y se perpetúa en este grupo de jóvenes.

A la vista de los datos parecería desde el punto de vista preventivo que aportar una mayor percepción acerca de los problemas ligados al consumo de drogas tiene su efecto pero no es suficiente; aportar información sobre los daños puede ser útil en algún momento, pero esta utilidad viene limitada una vez que los jóvenes vuelven a establecer su balance personal o colectivo sobre ventajas e inconvenientes; tratar de disminuir las ventajas que aportan los consumos de drogas podría ser una estrategia novedosa y útil, pero no es fácil transmitir con eficacia que una determinada conducta es menos beneficiosa de lo que se cree. Esto choca con una situación muy arraigada en nuestra sociedad que sitúa el conseguir placer como un valor de primer orden.

\section{REFERENCIAS}

Alarcão, M. (2000). (des) Equilíbrios familiares. Coimbra: Quarteto editora.

Andersson, B., Hibell, B., Beck, F., Choquet, M., Kokkevi, A., Fotiou, A. et al. (2007). Alcohol and Drug Use Among European 17-18 Year Old Students. Data from the ESPAD Project. Stockholm: The Swedish Council for Information on Alcohol and Other Drugs (CAN) and the Pompidou Group at the Council of Europe.

Bejerano, J., Lee, L. y Ugalde, F. (1999). Consumo de drogas y percepciones del riesgo derivado en estudiantes de universidades estatales de Costa Rica 1992-1996. Adicciones, 11, 269-278. 
Bellis, M., Hughes, K., Bennett, A., Thomson, R. (2003). The role of an international nightlife resort in the proliferation of recreational drugs. Adicciones, 98, 1713-1721.

Botella, J. (2000). Factores de riesgo y protección de carácter social relacionados con el consumo de drogas. Madrid: Ayuntamiento de Madrid. Área de servicios sociales. Plan municipal contra las drogas.

Calado, V. (2006). Drogas sintéticas. Mundos culturais, Música Trance e Ciberespaço. Lisboa: Núcleo de Investigação/ODT/IDT.

Calafat, A., Stocco, P., Mendes, F., Simon, J., Wijngaart, G., Sureda, M. et al. (1998). Characteristics and representation of ecstasy in Europe. Palma de Mallorca: Irefrea.

Calafat, A., Fernández, C., Bellis, M., Montserrat, J., Mendes, F., Hughes, K. et al. (2004). Cultural mediators in a hegemonic Nightlife. Palma de Mallorca: Irefrea España.

Camerotti, A. y Kornblit, A. (2005). Representaciones sociales y prácticas de consumo del éxtasis. Convergencia. 38, 312-333.

Castellana, M. y Lladó, M. (1999). Adolescencia y juventud: prevención y percepción del riesgo al consumo. Revista Española de Drogodependencias. 24, 27-33.

Castro, S. y Godoy, A. (1999). Relación entre dimensiones atribucionales referidas al consumo de tabaco y variables cognitivas y de personalidad. Revista Española de Drogodependencias. 24, 246-264.

Balsa, C., Farinha, T., Urbano, C. y Francisco, A. (2001). Inquérito nacional ao Consumo de Substâncias Psicoactivas na População Portuguesa. 2001. Lisboa: CEOS (Faculdade de Ciências Sociais e Humanas e Universidade Nova de Lisboa).

Costa, N. y Marques, R. (2002). Representações Mentais de Substancias Psicoactivas. Toxicodependências. 8, 3-12.

Fortin, M. (1999). O processo de investigação. Da concepção à realização. Loures : Lusociência.

Gallego, E., Hofstadt, C., Estévez, C., López, C. y Castillo, J. (2001), Representación social de la droga: posibilidad de aplicación práctica de un constructo eminentemente teórico.Salud y drogas. 1, 275- 286.

IDT (Instituto da Droga e da Toxicodependência) (2006) Relatório Anual 2005 - A situação do País em Matéria de Droga e toxicodependências. Vol. I. - Informação estatística - 2005. Lisboa: IDT.

Lomba, L. (2006). Consumo de ecstasy em jovens de Coimbra, In: Novas drogas e ambientes recreativos. (pp. 61-169). Coimbra: Lusociência.

López, G. (2007). La representación social de las drogas en un grupo de varones en reclusión en dos centros penitenciarios mexicanos. Anales de psicología. 23, 85-91.

Negreiros, J. (2000). Representações sociais acerca das drogas em crianças do $1 .^{\circ}$ ciclo do ensino básico: implicações para a prevenção. Toxicodependências. 6, 59-65.

Oliveira, A. (1998). Pertenças sociais e formas de percepção e representação da morte. Psicologia. 12, 115-137.

OEDT (Observatório Europeu da Droga e da Toxicodependência) (2001). Relatório Anual sobre a Evolução do Fenómeno da Droga na União Europeia: 2001. Luxemburgo: Serviço das Publicações Oficiais das Comunidades Europeias.

OEDT (Observatório Europeu da Droga e da Toxicodependência) (2002). Relatório Anual 2002 sobre a Evolução do Fenómeno da Droga na União Europeia e na Noruega. Luxemburgo: Serviço das Publicações Oficiais das Comunidades Europeias.

OEDT (Observatório Europeu da Droga e da Toxicodependência) (2006). Relatório Anual 2006. A Evolução do Fenómeno da Droga na Europa. Luxemburgo: Serviço das Publicações Oficiais das Comunidades Europeias.

Palmer, A., Montaño, J. J. y Calafat, A. (2000) Predicción del consumo de éxtasis a partir de redes neuronales artificiales. Adicciones, 12, 29-41

Peral, J. y Caballero-Reales, S. (1999). Estereotipos hacia los fumadores. Estudio de distintas variables en una muestra de adolescentes. Revista Española de Drogodependencias. 23, 265-283.

Rita, J. (2001). Prevenção de proximidade na sida e toxico-dependência. In: Presidência do conselho de ministros(Eds.), Regime geral da política de prevenção primária das toxicodependências. Documentos (Discussão Pública 18 de Junho - 10 de Agosto). Plano de acção nacional contra a droga e a toxicodependência - Horizonte 2004. (pp.33 - 36). Lisboa: IPDT.

Sierra, D., Pérez, M., Pérez A. y Nuñez, M. (2005). Representaciones sociales en jóvenes consumidores no consumidores de substancias psicoactivas. Adicciones. 17, 349-360.

SPTT (Serviço de Prevenção e Tratamento da Toxicodepêndencia) (2002). Informação geral para prevenção das toxicodependências. S.I.: Direcção Regional do Algarve do SPTT.

Vala, J. (1997). Representações sociais - para uma psicologia social do pensamento social. In: J. Vala \& M.B. Monteiro (Eds.), Psicologia Social. (pp. 353-384). Lisboa: Fundação Calouste Gulbenkian.

Valentim, A. (1998). Droga e toxicodependência nas representações de párocos e médicos. Análise Social.33, 55-90.

Vastag, B. (2001). Ecstasy experts want realistic messages. The Journal of the American Medical Association, 286, 777.

Zupiria, X., González, A. y Yllá, L. (1999). Cuestionario de creencias de los jóvenes sobre los efectos del alcohol. Revista de Psiquiatría. 26, 1-8. 